\title{
DNA ploidy, cell proliferation, and HIV/EBV association in Tanzanian malignant lymphomas
}

\author{
Amos Mwakigonja ${ }^{1,2^{*}}$, German Wannhoff ${ }^{2}$, Thomas Heiden ${ }^{3}$, Anna Porwit ${ }^{4}$, Peter Biberfeld ${ }^{2}$, Ephata Kaaya ${ }^{1,2}$ \\ From $12^{\text {th }}$ International Conference on Malignancies in AIDS and Other Acquired Immunodeficiencies \\ (ICMAOI) \\ Bethesda, MD, USA. 26-27 April, 2010
}

\begin{abstract}
Background
Malignant lymphomas (ML) are increasingly important causes of morbidity and mortality in sub-Saharan Africa including Tanzania, possibly due to HIV and AIDS. However, the biological characterization ML including their HIV and Epstein-Barr virus (EBV) association as well as DLBCL subtypes in Tanzania is still sketchy. This prevents diagnostic/prognostic comparison as well as application of established therapeutic protocols.
\end{abstract}

\section{Materials and methods}

Selected archival, diagnostic ML biopsies $(\mathrm{N}=60)$ collected at Muhimbili National Hospital (MNH), Dar es Salaam, Tanzania, between 1996 and 2006 and their corresponding clini$\mathrm{cal} /$ histopathological notes were analyzed by histopathology; immunohistochemistry (IHC) using CD20, CD3, CD30, CD10, MUM1p, BCL-6, BCL-2, and Ki-67 cell markers; flow-cytometry (FC) for DNA ploidy; and in situ hybridization (ISH) $(n=25)$ for EBV-encoded RNA (EBER). Available sera $(\mathrm{N}=35)$ were screened by ELISA for HIV antibodies.

\section{Results}

Out of the evaluated cases, 27 were diffuse large B-cell lymphoma (DLBCL) of which a slight majority $(55.6 \%$, $\mathrm{n}=15 / 27)$ had activated B cell-like (ABC) and $44.4 \%$ $(12 / 27)$ had germinal center B cell-like (GCB) immunophenotype, although this was not statistically significant (p-value 0.547, Chi ${ }^{2}$ Test). Overall, 40\% (24/60) ML were aneuploid mostly $(63.0 \%, 17 / 27)$ the DLBCL and T-cell lymphoma (TCL) [54.5\%, 6/11] which differences were not statistically significant (p-value $0.06, \mathrm{Chi}^{2} \mathrm{Test}$. DNA index (DI) of FC-analyzed ML ranged from 1.103 to 2.407

\footnotetext{
*Correspondence: rodgeramos@yahoo.com

${ }^{1}$ Department of Pathology, Muhimbili University of Health and Allied

Sciences, Dar es Salaam, Tanzania

Full list of author information is available at the end of the article
}

(median=1.51) and most $(75.0 \%)$ aneuploid cases showed high $(>40 \%)$ cell proliferation by Ki-67 reactivity (p-value 0.031 , statistically significant, Fisher's Exact Test). The majority $(51.4 \%, 19 / 37)$ of EBER ISH analyzed lymphoma biopsies were positive (p-value 0.87 , not statistically significant, $\mathrm{Chi}^{2}$ Test). Of the serologically tested lymphomas $40.0 \%(14 / 35)$ were HIV positive, mostly with high $(\geq 40.0 \%) \mathrm{Ki}-67$ reactivity [p-value $=0.05$, statistically significant, Pearson Correlation].

\section{Conclusions}

Lymphomas at $\mathrm{MNH}$ appear to have frequent aneuploidy and EBER positivity as well as high DNA indices and tumor proliferation (Ki-67). DLBCL phenotype heterogeneity was similar to that observed in other countries suggesting applicability of established intervention approaches. HIV was apparently associated with high lymphoma cell proliferation but extended studies are needed to clarify this.

\section{Acknowledgements}

This article has been published as part of Infectious Agents and Cancer Volume 5 Supplement 1, 2010: Proceedings of the $12^{\text {th }}$ International Conference on Malignancies in AIDS and Other Acquired

Immunodeficiencies (ICMAOI). The full contents of the supplement are available online at http://www.biomedcentral.com/1750-9378/5? issue=S1.

\section{Author details}

${ }^{1}$ Department of Pathology, Muhimbili University of Health and Allied Sciences, Dar es Salaam, Tanzania. ${ }^{2}$ Department of Oncology-Pathology, Karolinska University Hospital, Solna/Karolinska Institute, Stockholm, Sweden. ${ }^{3}$ Otto-Heubner-Center for Pediatrics, Charité Campus, Virchow-Klinikum, Berlin, Germany. ${ }^{4}$ Department of Pathology, Radiumhemmet, Karolinska University Hospital, Solna, Stockholm, Sweden.

Published: 11 October 2010

doi:10.1186/1750-9378-5-S1-A21

Cite this article as: Mwakigonja et al:: DNA ploidy, cell proliferation, and HIV/EBV association in Tanzanian malignant lymphomas. Infectious Agents and Cancer 2010 5(Suppl 1):A21. 